\title{
Long-Term Optical Imaging and Spectroscopy Reveal Mechanisms Underlying the Intrinsic Signal and Stability of Cortical Maps in V1 of Behaving Monkeys
}

\author{
Eran Shtoyerman, Amos Arieli, Hamutal Slovin, Ivo Vanzetta, and Amiram Grinvald \\ Department of Neurobiology, The Weizmann Institute of Science, 76100 Rehovot, Israel
}

\begin{abstract}
Explorations of learning and memory, other long-term plastic changes, and additional cognitive functions in the behaving primate brain would greatly benefit from the ability to image the functional architecture within the same patch of cortex, at the columnar level, for a long period of time. We developed methods for long-term optical imaging based on intrinsic signals and repeatedly visualized the same functional domains in behaving macaque cortex for a period extending over 1 year. Using optical imaging and imaging spectroscopy, we first explored the relationship between electrical activity and hemodynamic events in the awake behaving primate and compared it with anesthetized preparations. We found that, whereas the amplitude of the intrinsic signal was much larger in the awake animal, its temporal pattern was similar to that observed in the anesthetized animals. In both groups, deoxyhemoglobin concentration reached a peak 2-3 sec after stimulus onset. Furthermore, the early activity-
\end{abstract}

dependent increase in deoxyhemoglobin concentration (the "initial dip") was far more tightly colocalized with electrical activity than the delayed increase in oxyhemoglobin concentration, known to be associated with an increase in blood flow. The implications of these results for improvement of the spatial resolution of blood oxygenation level-dependent functional magnetic resonance imaging are discussed. After the characterization of the intrinsic signal in the behaving primate, we used this new imaging method to explore the stability of cortical maps in the macaque primary visual cortex. Functional maps of orientation and ocular dominance columns were found to be stable for a period longer than 1 year.

Key words: anesthesia; behaving monkeys; brain mapping; cortical columns; f-MRl; hemodynamics; optical imaging; plasticity; primary visual cortex
Cortical studies of anesthetized mammals have contributed profoundly to our understanding of cortical functions at the level of single neurons and cortical columns (Mountcastle, 1957; Hubel and Wiesel, 1962, 1969). However, anesthetized subjects are unsuitable for many types of studies, such as motivation, attention or arousal affecting sensory processing and perception, motor function, consciousness, and other cognitive functions. In addition, long-term plastic changes related to memory and learning or recovery of function after trauma or stroke are difficult to pinpoint without imaging; it may be like a search of a needle in a haystack. Studies in human subjects are limited to noninvasive examinations; electrical recording or anatomical techniques are not an option. Therefore, for the foreseeable future, the awake monkey model is likely to remain the preparation of choice for research at the level of single cells and cortical columns.

Optical imaging based on intrinsic signals (Grinvald et al., 1986; Frostig et al., 1990; Ts'o et al., 1990) is currently the tool of choice for obtaining high-resolution functional maps of the cortex (Bonhoeffer and Grinvald, 1991, 1996; Shmuel and Grinvald, 1996; Wang et al., 1996; Bosking et al., 1997; Shoham et al., 1997) (for review, see Grinvald et al., 1999). Furthermore, to our knowledge, it is the only tool currently available to reveal the relationships between a few different functional maps within one area (Bartfeld and Grinvald, 1992; Hubener et al., 1997). This method, however, was limited to one recording sessions from each anesthetized monkey or to a few short recording sessions from behaving monkeys (Grinvald et al., 1991; Vnek et al., 1999). Our goal was to

\footnotetext{
Received Feb. 17, 2000; revised Aug. 17, 2000; accepted Aug. 17, 2000.

This work was supported by grants from the Israeli-German Research Program, the German Israeli Foundation, and a grant from the Horace H. Goldsmith Foundation. We thank Chaipi Wijnbergen, Yuval Toledo, and Dov Ettner for technical assistance. Correspondence should be addressed to Amiram Grinvald, Department of Neurobiology, The Weizmann Institute of Science, 76100 Rehovot, Israel. E-mail: amiram.grinvald@weizmann.ac.il.

Copyright (C) 2000 Society for Neuroscience $0270-6474 / 00 / 208111-11 \$ 15.00 / 0$
}

remove these limitations by developing methods for repeated, long-term imaging from behaving animals.

The first topic we address here is related to the basics of functional brain imaging by either optical imaging or functional magnetic resonance imaging (f-MRI). It has been assumed that optical imaging based on intrinsic signals (Frostig et al., 1990; Malonek and Grinvald, 1996; Vanzetta and Grinvald, 1999; Grinvald et al., 2000) and blood oxygenation level-dependent (BOLD) f-MRI (Ogawa et al., 1990, 1992; Kwong et al., 1992) rely on similar activity-dependent signals originating from blood microcirculation. However, the intrinsic signals measured by these two imaging methodologies have different time courses. In particular, the early component referred to as the "initial dip" has rarely been detected by low magnetic field strength f-MRI. Here we present evidence that this difference in time courses cannot be explained by species differences (i.e., monkey vs cat) or the state of anesthesia (i.e., awake animal vs anesthetized one). In fact, recently the initial dip was detected with high magnetic field strength (Menon et al., 1995; Hu et al., 1997; Kim et al., 2000a,b; Logothetis et al., 1999, 2000).

The second topic we studied using the newly developed methods was the stability of functional maps in the cortex of adult monkeys. In many studies of plasticity, the stability of functional maps in adult animals under normal conditions has been assumed but never fully demonstrated. A few studies have shown that adults animals do not show changes in certain statistical properties, such as the relative size of the ocular dominance columns (LeVay et al., 1980) or the fraction of neurons responding to lines of a given orientation (Creutzfeldt and Heggelund, 1975). Others have demonstrated the stability of gross retinotopic or somatosensory organization (Merzenich et al., 1984; Kaas et al., 1990; Heinen and Skavenski, 1991). Before optical imaging was introduced, however, comparisons of the detailed structure of functional maps over periods of weeks and months were difficult to achieve. With the availability of optical imaging of intrinsic signals, a closer study of map stability became possible. Kim and Bonhoeffer (1994), Godecke and Bonhoeffer (1996), and Chapman et al. (1996) demonstrated the stability of 

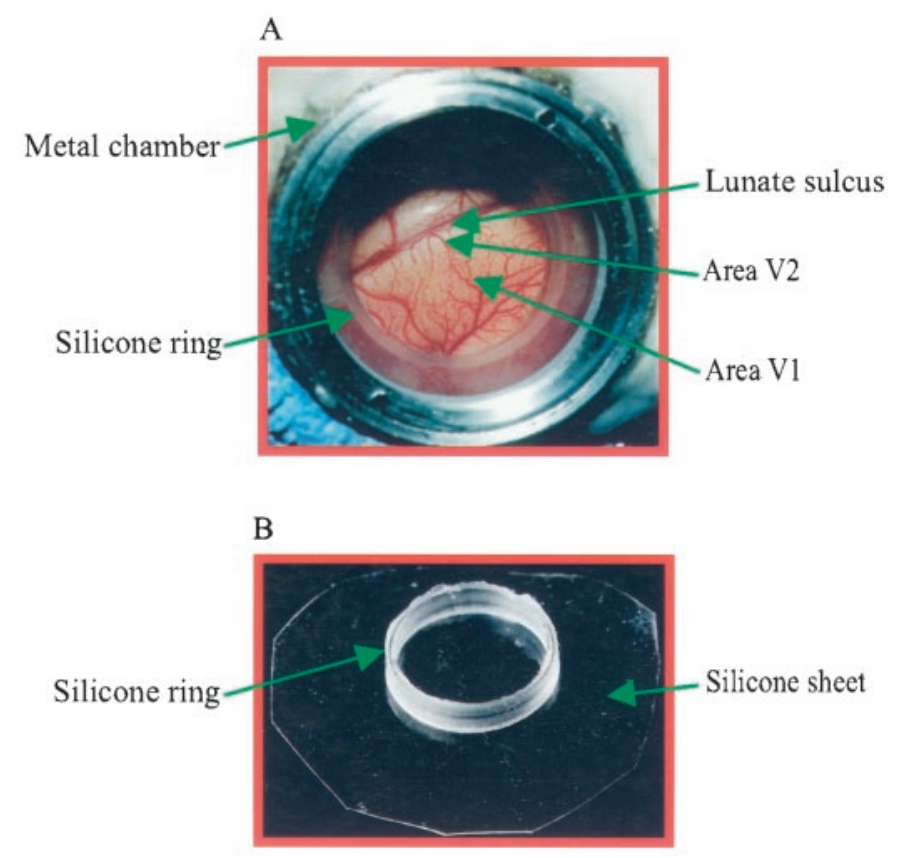

Figure 1. Cranial window and artificial dura mater. $A$, The exposed cortex as seen through the transparent silicon membrane. The lunate sulcus is in the top left part of the cranial window. V1 and V2 are thus available for optical imaging. This picture was taken 5 months after insertion of the artificial dura over the exposed cortex. $B$, Enlargement of the artificial silicon dura in the cranial window shown in $A$. The silicon ring in the center stabilizes the artificial dura and prevents the real dura from growing on the imaging area.

orientation maps in the developing visual cortex of kittens and young ferret over a period of several weeks. In the adult rat, Masino and Frostig (1996) found a size variability of as much as $48 \%$ over a period of months in the representation of whiskers in rats' barrel cortex. These authors showed that, even without any change in the rats' environment, the maps in a primary sensory area showed large ongoing fluctuations. Much larger plasticity in the cortical representation of a single whisker has been detected by long-term optical imaging performed by the same group (Polley et al., 1999), after the innocuous removal of neighboring whiskers. Are there changes in cortical maps, over a time scale of months, in the major columnar systems in the primary visual cortex of primates? Although the answer to this question is crucial for studies of plasticity in adult primates brain, maps in the primate have never been monitored for longer than couple of weeks. The present study demonstrates that the recently developed optical imaging techniques now make it possible to study either the stability or the plasticity of cortical maps in adult primates over a period longer than 1 year.

Parts of this work have been published previously in abstract form and methodological review (Shtoyerman et al., 1995, 1998; Bonhoeffer and Grinvald, 1996; Slovin et al., 1999).

\section{MATERIALS AND METHODS}

Animals. Two adult Macaca fascicularis monkeys and four adult Macaca mulatta monkeys were used for the study on behaving animals. Ten adult Macaca fascicularis monkeys were used in the development of the technique of long-term recording from chronic, anesthetized animals. The surgical and recording procedures in acute anesthetized animals have been described in detail previously (Ts'o et al., 1990; Grinvald et al., 1991, 1999). Here we describe only the surgical procedure used to prepare the monkeys for repeated recordings while they are awake and behaving.

Head holder and chambers for optical recording. All surgical procedures were performed according to the National Institutes of Health guidelines. The animals were first anesthetized with a mixture of ketamine $\mathrm{HCl}$ (10 $\mathrm{mg} / \mathrm{kg})$ and xylazine $(1 \mathrm{mg} / \mathrm{kg})$ and then transferred to the operating room, where they were intubated, provided with an intravenous catheter, anesthetized with $1 \%$ isoflurane in a $3: 2$ mixture of $\mathrm{NO}_{2} / \mathrm{O}_{2}$, and artificially respirated. The state of the animals and the level of anesthesia were monitored continuously by measurement of EEG, electrocardiogram, endtidal $\mathrm{CO}_{2}$, and rectal temperature. A 4-cm-long head holder was cemented to the cranium with dental acrylic cement. Two chambers (inner diameter of $25 \mathrm{~mm}$ ) (Fig. $1 A$ ) for optical imaging were placed on the posterior part of the skull over the primary visual cortex. A few stainless steel screws were inserted into the skull around the two cranial windows, and a $0.7 \mathrm{~mm}$ stainless steel wire was wrapped around the screws for additional support. A dental acrylic cap was then built around the optical chamber and the head holder. Appropriate analgesics (oral dipyrone, paracetamol, or flunixine) and preventive antibiotics (usually a daily intramuscular injection of $20-30 \mathrm{mg} / \mathrm{kg}$ cefonicid) were given postoperatively. After a 1 week of recovery period, the monkeys were trained for at least 1 month on visual fixation tasks.

Cortex exposure. After the recovery period, the monkeys underwent a second surgical procedure in which the cortex was exposed. The operation was conducted under strictly sterile conditions to minimize the risk of infection The induction of anesthesia was as above, but thiopental sodium was preferred to isoflurane because it reduces the risk of brain swelling $\left(10-20 \mathrm{mg} / \mathrm{kg}\right.$, followed by continuous infusion of $\left.1-3 \mathrm{mg} \cdot \mathrm{kg}^{-1} \cdot \mathrm{hr}^{-1}\right)$. Before the sterile procedure was started, the monkeys were shaved and cleaned thoroughly with two antiseptic solutions (10\% povidone-iodine and $0.5 \%$ chlorhexidine). Using a trephine, a 23 -mm-diameter craniotomy was performed inside one of the implanted chambers. To allow insertion of an artificial dura under the real dura mater without damage to the cortex, as described below, it was important to avoid any swelling of the brain. We used a few techniques to achieve this: (1) $30 \mathrm{~min}$ before opening of the dura, mannitol $(500 \mathrm{mg} / \mathrm{kg}$ ) was inf used intravenously over a period of 10 min; (2) 15 min before opening of the dura, intravenous furosemide (1-3 $\mathrm{mg} / \mathrm{kg}$ ) was slowly administered; (3) the monkey was hyperventilated so that the end-tidal $\mathrm{CO}_{2}$ level was $26 \mathrm{mmHg}$; (4) a few minutes before opening of the dura, the monkey was given a large dose of thiopental sodium $(5 \mathrm{mg} / \mathrm{kg}$ over $5 \mathrm{~min}$ ); and (5) the body was lowered relative to the head.

Resection of the dura was started only when the cortex was not exerting any pressure on it, as judged from the free movement of the dura mater, accompanying each respiratory cycle. Initially, we cut only the upper layers of the dura, including the layer containing the blood vessels, in an $\mathrm{X}$-shaped manner. We then waited until bleeding from the superficial cut ceased. This was done to avoid any contact between blood and cortex, because we have some indications that there are at least short-term effects of blood on cortical functions (A. Grinvald, unpublished results). When the bleeding had stopped, the four "leaves" of the dura were separated, and each leaf was pulled up and glued to the bone using tissue adhesive (Histoacryl tissue adhesive; Braun, Melsungen, Germany). The exposed cortex was then gently flushed with artificial CSF (ACSF) (Wilson et al., 1978) at body temperature. Acepromazine or flumixin were used for analgesia.

Artificial dura. Covering of the cortex immediately after its exposure with an artificial dura turned out to be crucial for preventing infections and maintaining the cortex in good shape for long periods. We used a homemade artificial dura mater, molten in one piece, consisting of a transparent silicon rubber sheet, $32 \mathrm{~mm}$ in diameter and $0.2-\mathrm{mm}$-thick, attached to a silicon ring, 16-19 $\mathrm{mm}$ in outer diameter, 1-mm-thick, and 4-mm-high (Fig. $1 B$ ). The edges of the silicon sheet were inserted under the bone and the real dura. To "seal" the cortex back, as it had been when it was covered by its own natural dura, and to prevent adhesion of the cut dura to the cortex, at least $4 \mathrm{~mm}$ of the sheet were inserted between the dura and the cortex. When the artificial dura was in its proper position, the cut edge of the natural dura enfolded the silicon ring in the middle of the sheet, stabilizing it and preventing the natural dura from growing into the central area and covering it. Thus, the cortex remained perfectly visible for $>1$ year (Fig. $1 A$ ). Within a few weeks after the operation, the dura had grown around the ring, thus creating a good seal of the cortex. In a few cases, traces of blood that remained between the silicon sheet and the cortex initially reduced the visibility of the cortex. These traces disappeared within a few days, however, and the surface of the cortex remained clearly visible (Fig. $1 A$ ).

Another advantage of using this artificial dura is that it proved easy to penetrate it with electrodes. The clear visibility of the cortex facilitated targeted electrical recordings of various types, targeted microstimulation, or targeted tracer injections. The artificial dura is available on request. Additional details on the artificial dura and the optical chamber for manipulatable electrical recordings will be described in detail elsewhere (A. Arieli and A. Grinvald, unpublished).

After insertion of the artificial dura, the chamber was filled with a solution of $1 \%$ agarose, $10 \mathrm{mg}$ of neomycin, $20,000 \mathrm{U}$ of polymyxin $\mathrm{B}, 2 \mathrm{mg}$ of dexamethasone sodium phosphate, and ACSF. The solution was introduced only after being cooled to $39^{\circ} \mathrm{C}$ so that it solidified immediately after filling the chamber. A glass cover sealed the chamber, and a metal cover above it prevented accidental damage to the cortex. Postoperatively, oral Augmentin (amoxycillin and clavulanic acid; Beecham Research Laboratories, Brentford, UK) or a mixture of ampicillin and aztreonam was injected intramuscularly as preventive antibiotics. The antibiotics were administered for 7-14 d.

Routine treatment. During periods of imaging, the chamber was opened between one to three times per week for cleaning, which was done in an operating room under strictly sterile conditions. The monkeys were awake during this painless procedure. In each treatment, the fluid in the chamber above the silicon sheet was sampled for bacteriological and mycological examination. This frequent examination was found to be crucial because some type of infections that develop on the dura (mostly infections by 
Staphylococcus aureus or Enterococcus species) might penetrate the cortex within $2-4 \mathrm{~d}$ if no proper treatment is used. The fluid in the chamber was then replaced by a new mixture of ACSF, agarose (0.3-3\%), dexamethasone $(0.2 \mathrm{mg} / \mathrm{ml})$, and a cocktail of antibiotics and anti-fungal drugs, according to the microbiology result of the previous fluid sampling. Among the ingredients usually included in the cocktail were as follows: chloramphenicol $(1 \mathrm{mg} / \mathrm{kg})$, polymyxin $\mathrm{B}(2000 \mathrm{U} / \mathrm{ml})$, neomycin $(0.5 \mathrm{mg} / \mathrm{ml})$, gentamycin $(0.5 \mathrm{mg} / \mathrm{ml})$, streptomycin $(1 \mathrm{mg} / \mathrm{ml})$, bacitracin $(500 \mathrm{U} / \mathrm{ml})$, vancomycin $(0.3 \mathrm{mg} / \mathrm{ml})$, ceftazidime $(2 \mathrm{mg} / \mathrm{ml})$, ceftriaxone $(2 \mathrm{mg} / \mathrm{ml})$, ofloxacin $(3 \mathrm{mg} / \mathrm{ml})$, and amphotericin $(1 \mathrm{mg} / \mathrm{ml})$. The antibiotics gentamycin and neomycin were administered with care, because they are known to cause epileptic seizures at high concentration.

A high concentration of agarose was necessary to prevent quick regrowth of the natural dura. To obtain good visibility of the exposed cortex through the agarose solution, we filled the chamber in two stages. First, we applied a thin layer of concentrated solution of agarose (3\%) and antibiotics onto the dura and the bone but not on the imaging area of the exposed cortex. Second, we filled the whole chamber with a dilute solution of agarose $(0.3 \%)$ and colorless antibiotics. In the rare cases when an infection of the dura or the cortex was found during the cleaning procedure, the monkey was given systematic antibiotic treatment with one or more of the following antibiotics: gentamycin, cefazolin, ceftazidime, ceftriaxone, aztreonam, ampicillin, amoxycillin, cloxacillin, and ofloxacin. The mixture of antibiotics was chosen according to the result of a bacteriological tolerance test done on the sample taken during the cleaning procedure. Usually 2 weeks of systematic treatment with an appropriate mixture of antibiotics exterminated the infection.

Optical imaging. The procedure of optical imaging in anesthetized animals has been reviewed in detail previously (Bonhoeffer and Grinvald, 1996; Grinvald et al., 1999). Here we describe the imaging procedure for the behaving monkeys only.

Before the imaging session, the monkey was placed in a primate chair, which was attached by means of a few rigid metal bars to a heavy table to reduce movement noise. The monkey's head was fixed to the metal bars at two points, one connected to the middle of the skull and the other on the posterior part of the head close to the chamber. The metal cover protecting the glass of the cranial window was removed to expose the transparent chamber window. A sensitive CCD video camera, equipped with a tandemlens arrangement (Ratzlaff and Grinvald, 1991), was mounted over the window of the transparent chamber. The camera was part of an imaging system (Imager 2001; Optical Imaging Inc., Germantown, NY) that provides a digitized picture at a resolution of $756 \times 574$ pixels. The system also provides a real-time differential picture that allows continuous monitoring of minor changes, reflecting respiration and heartbeat pulsations or $0.1 \mathrm{~Hz}$ vasomotion noise, in the cortical image. This was especially important during the spectroscopic measurements. The exposed cortex was illuminated using two light guides fixed to the metal bars. Before starting the functional mapping, we photographed the cortex while illuminating it with green light $(540 \mathrm{~nm})$ to emphasize the vascular pattern. Images were then recorded, and the vascular pattern was used to focus the camera onto the appropriate region of the exposed cortex and to align the camera such that the imaging plane would be parallel to the cortical surface. Once an optimally focused image was obtained, the camera was lowered by $400 \mu \mathrm{m}$ to reduce blood vessel artifacts. The camera was then connected to the head holder at two points to further reduce any movement of the skull relative to the camera itself.

During the imaging session, interference filters were used to select the desired wavelength for imaging. A filter of $605 \mathrm{~nm}$ (bandwidth of $10 \mathrm{~nm}$ ) usually gave the best signal-to-noise ratio. The images of the cortex were digitized at video rates over a period of $6-15 \mathrm{sec}$. The data were compressed by summing several video frames, thus reducing the time resolution to $300-500 \mathrm{msec}$ per stored frame.

Spectroscopy. The optical imaging spectroscopy procedures were similar to those described previously (Malonek and Grinvald, 1996; Malonek et al., 1997). Briefly, images of the exposed cortex were obtained using an optical apparatus composed of two tandem-lens macroscopes (Fig. 2). The apparatus formed two image planes. An opaque disk with a transparent slit was inserted into the position of the first image plane so that only the light from the slit would be transferred. The macroscope was aligned so that the slit would be above an area of the cortex that exhibits clear ocular dominance columns and parallel to the cortical surface, as well as perpendicular to the columns. The light emerging from the slit was dispersed by a diffraction grating positioned between the objective and the imaging lens of the second tandem-lens macroscope. The grooves of the grating were parallel to the slit, so that the light coming from every point along the slit was separated into its spectral components along the dimension perpendicular to the slit. Thus, the picture generated in the second image plane contained spectral information along the dimension perpendicular to the slit and spatial information along the dimension parallel to the slit (Fig. 3). Two light guides were used to illuminate the cortex. The light source was a halogen lamp. Using suitable optical filters, we adjusted the spectral profile of the light to make the light spectrum as flat as possible in the range of 530-650 nm. A sensitive CCD camera (part of the Imager 2001 system) recorded the image from the second image plane.

Behavioral paradigm and visual stimuli. Monkeys were trained to perform a variety of visual-auditory paradigms, but only the simplest paradigms were used in this study. A typical task started when the monkey fixates on

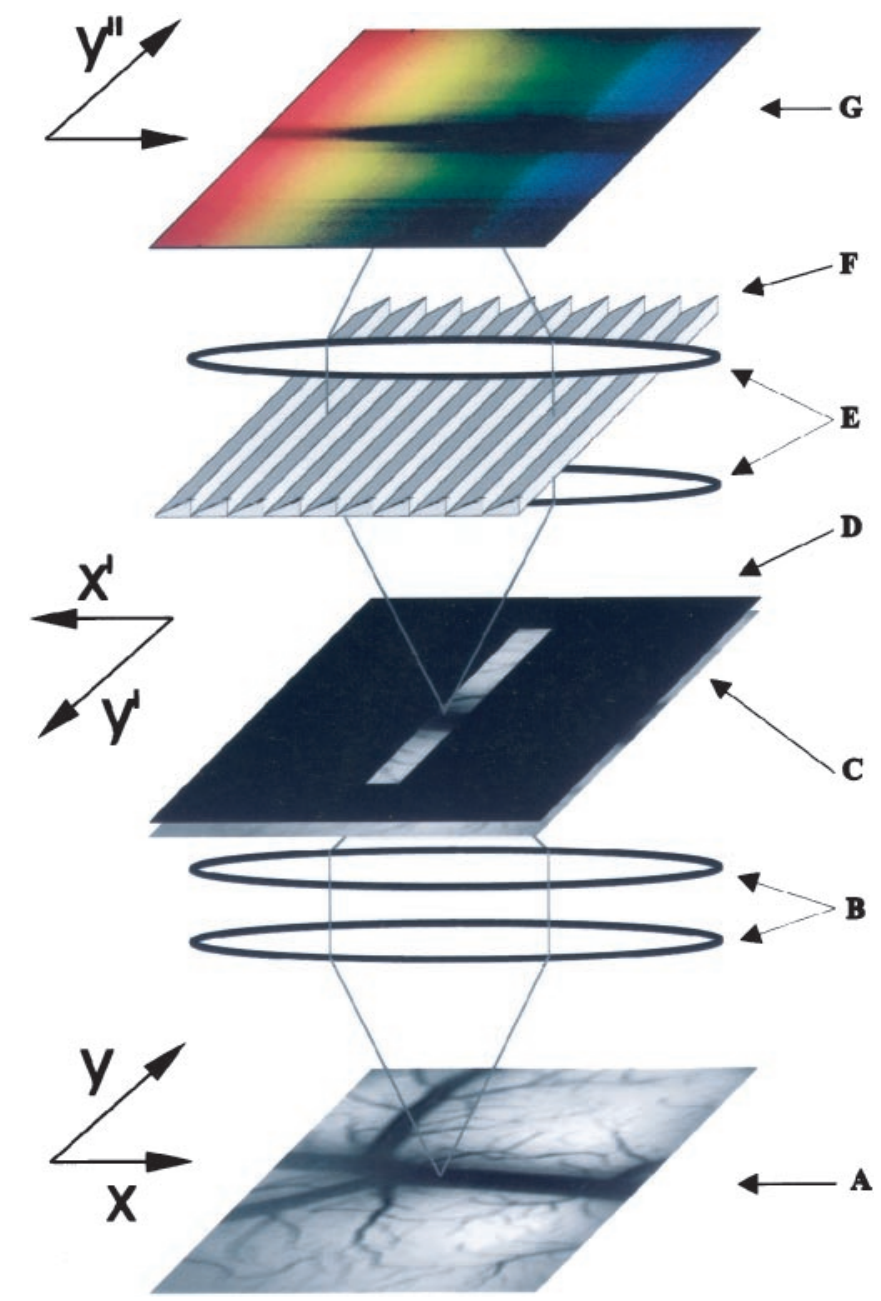

Figure 2. Scheme of the imaging spectroscope (adapted from Malonek et al., 1996). The bottom tandem-lens macroscope $(B)$ creates an image of the exposed cortex $(A)$ at the first image plane $(C)$ in which an opaque disk with a transparent slit is positioned $(D)$. The image of the slit is collimated by the objective of the second tandem-lens macroscope $(E)$, diffracted along the axis perpendicular to the slit by the diffraction grating $(F)$, and focused on the camera $(G)$.

a small dot $\left(0.1 \times 0.1^{\circ}\right)$ displayed on a cathodic ray tube screen. Four seconds later, a stimulus appeared on the screen. The stimulus, usually a moving square grating (contrast, $50 \%$; size, $10 \times 15^{\circ}$; spatial frequency, $0.5-2$ cycles per degree; temporal frequency, $2-5 \mathrm{~Hz}$; orientation, $0,45,90$, or $135^{\circ}$ ) was displayed for various durations. Computer-controlled shutters in front of the eyes allow either binocular or monocular stimulation. The monkey had to keep fixating on the small dot until a small change occurs in the intensity of the fixation point, 3-9 sec after the stimulus has disappeared. To earn a reward (a drop of juice), the monkey must move its hand within $500 \mathrm{msec}$ from the change. To allow for the relaxation of activity-dependent vascular changes, the stimulus was followed by an intertrial interval of $6 \mathrm{sec}$, during which time the screen was dark and there was no light in the room. Two different control conditions were used: in the first, the stimulus consisted of a fixation dot with no grating, and in the second, both eyes were covered with eye shutters.

We used a personal computer equipped with a Sergeant Pepper Plus board (Number Nine, Lexington, MA) and a Keithley 575 system (Keithley Instruments, Cleveland, $\mathrm{OH}$ ) to generate stimuli and to control the experiment. Eye movements were monitored with a Dr. Bouis infrared oculometer (Dr. Bouis Devices, Karlsruhe, Germany). Stimuli were displayed on a 21 inch Mitsubishi monitor, at $60 \mathrm{~Hz}$, placed $100 \mathrm{~cm}$ from the monkey.

Data acquisition. A reference frame was collected once for each set of stimuli. The Imager 2001 system subtracted this frame in real time from each of the raw video frames received from the camera afterward and amplified the result further before it was digitized. An automatic rejection procedure was applied in the experiments with the behaving monkey: exceptionally noisy trials were excluded from the calculation of on-line maps. A trial was considered noisy if the mean difference between two subsequent frames was higher than a predefined threshold. In most experiments, we selected a threshold that led to the rejection of $10 \%$ of the trials 
A

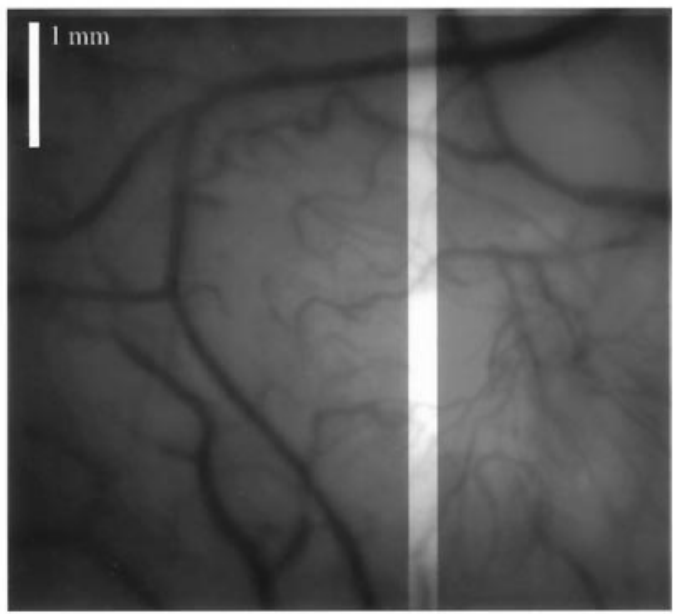

Slit
B

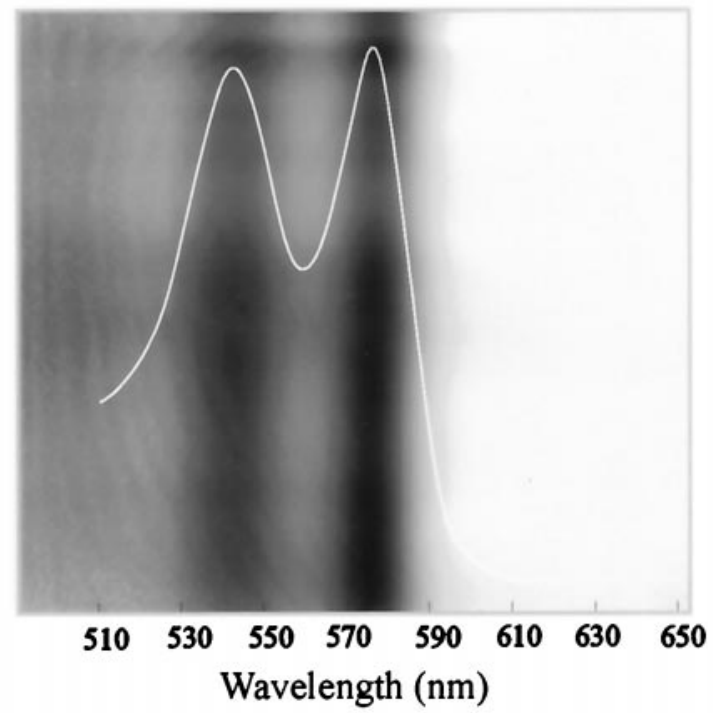

$\mathrm{D}$

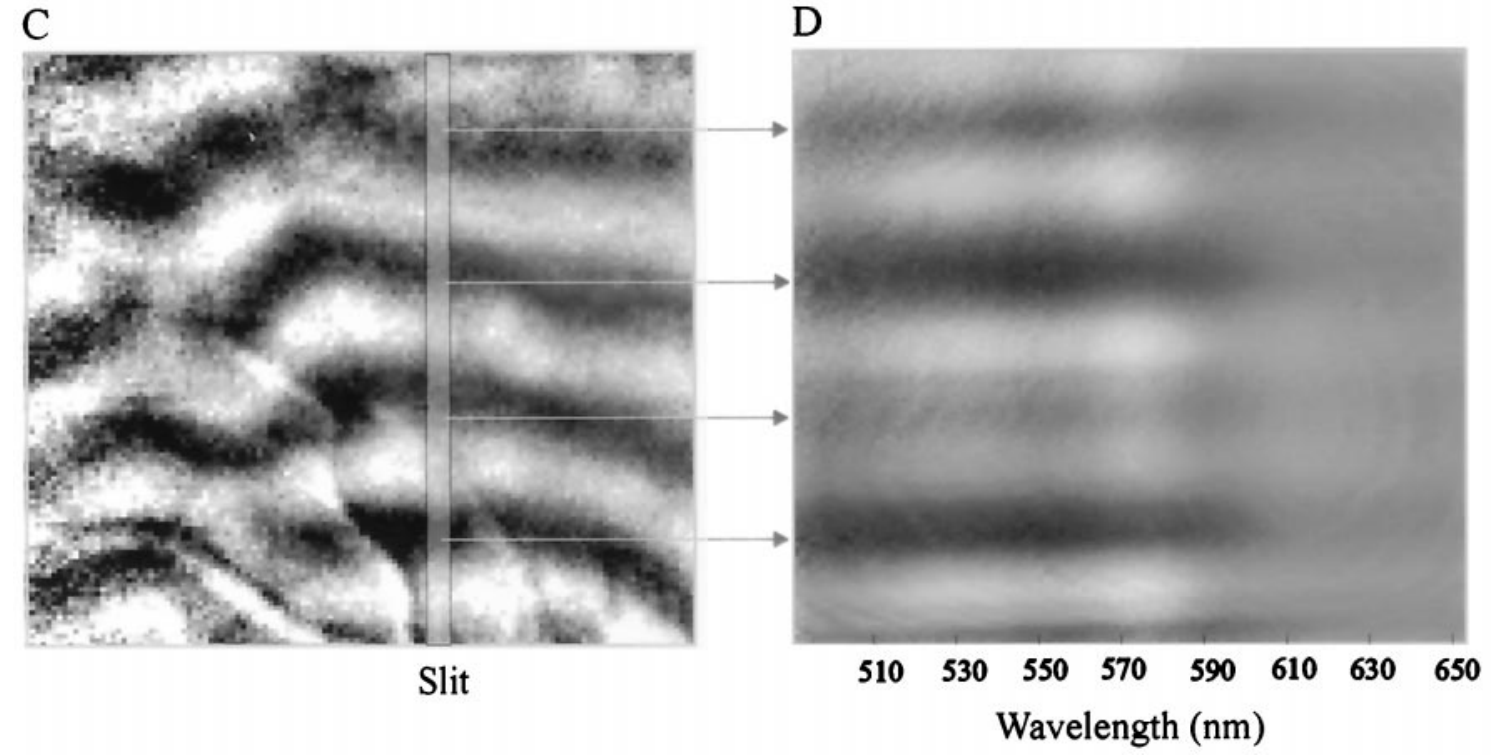

Figure 3. Example of an image recorded by the optical imaging spectroscope. $A$, Cortical surface illuminated with green light (570 nm) to emphasize the blood vessels. The location of the slit is highlighted. $B$, An example of a spatiospectral image obtained by the macroscope. The raw pictures were imaged through the slit while the cortex was illuminated with broad-spectrum light. The image shown here was obtained by subtracting a frame collected before stimulus onset from a frame collected $5 \mathrm{sec}$ after stimulus onset. The $x$-axis is the spectral dimension, and the $y$-axis is the spatial dimension along the slit. The $t w o$ dark vertical bands in the image correspond to the two absorption peaks of oxyhemoglobin at 540 and $580 \mathrm{~nm}$, as can be seen from the absorption spectrum of oxyhemoglobin (plotted in white). C, Ocular dominance map obtained through the spectroscope without the slit, at a wavelength of $605 \mathrm{~nm}$. The location of the slit during the subsequent imaging spectroscopy session is marked on the map. $D$, Example of a spatiospectral ocular dominance map. The map was obtained through a slit located in the position shown in $C$. The arrows show the correspondence between the spatiospectral bands and the two-dimensional ocular dominance stripes.

containing the largest noise. This rejection procedure was necessary, because a single trial containing exceptionally large noise from a sudden movement of the monkey could readily destroy the quality of accumulated images averaged over 10-20 noise-free trials (Grinvald et al., 1991).

Data analysis. Functional maps were derived from the raw data by summing the chosen frames from one or more stimuli and then dividing (a division is equivalent to subtraction) (Bonhoeffer and Grinvald, 1996) the result by the sum of the chosen frames from other stimuli. As an example, to obtain a map of ocular dominance columns, we summed all of the frames collected when the monkey's left eye was open and divided the resulting image by the sum of all the frames collected when the right eye was open. The resulting map was then displayed on the Imager 2001 monitor.

The first stage in the analysis of the spectroscopic data were similar to the above procedure, i.e., the images obtained when the monkey was stimulated were divided by the images obtained when it was unstimulated. The intensity of the pixels along horizontal lines (perpendicular to the slit) in the resulting pictures thus represents changes in the reflection spectrum profile attributable to the stimulation. To extract the changes in oxyhemoglobin and deoxyhemoglobin concentrations from the differential reflection spectrum, we used the following linear approximation:

$$
\Delta R(\lambda, t) \approx K_{o}(t) \cdot \varepsilon_{o}(\lambda)+K_{d}(t) \cdot \varepsilon_{d}(\lambda)+\mathrm{LS}(t)
$$

where $\Delta R$ is the change in the reflected light, $K_{O}$ is an approximate linear function of the oxyhemoglobin concentration, $\epsilon_{o}$ is the extinction coefficient of oxyhemoglobin, $K_{d}$ and $\epsilon_{d}$ are the corresponding parameters for deoxyhemoglobin, and LS is a wavelength independent term encoding light-scattering effects. We used linear regression to determine the values of $K_{O}$ and $\mathrm{K}_{d}$ that would best fit the observed spectrum in each frame and in each spatial location.

\section{RESULTS}

\section{Comparison between awake monkeys and anesthetized monkeys}

Identical stimuli were used for the awake and the anesthetized monkeys to allow a detailed comparison of the corresponding optical signals. The stimuli were optimized for activating the primary visual area (high-contrast moving square grating of $10 \times 15^{\circ}$ 


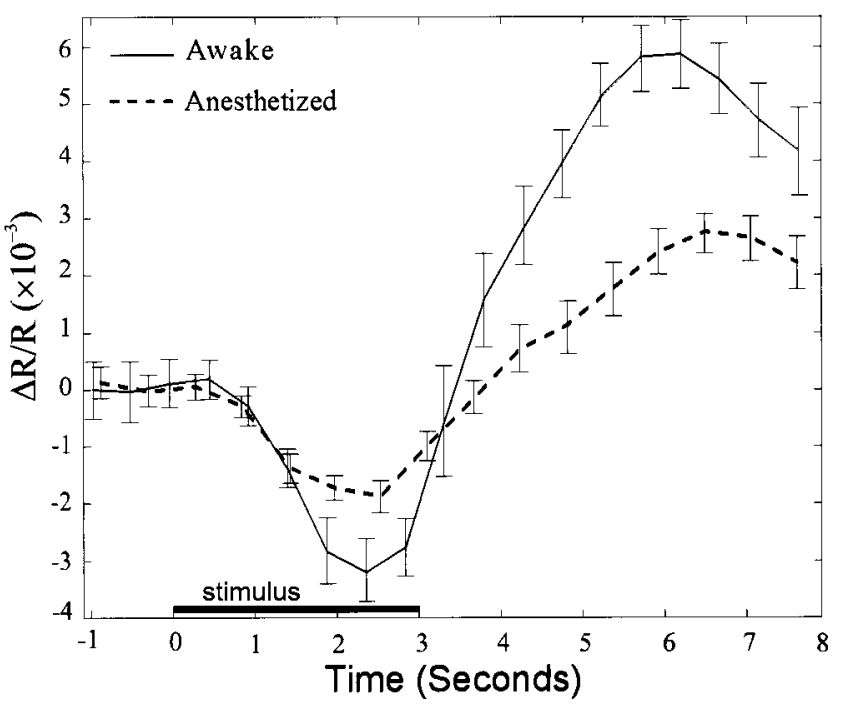

Figure 4. Time course of the global signal. The intensity of the reflected light during the stimulus condition relative to the intensity during the blank condition is plotted as a function of time from stimulus onset for awake (solid line) and anesthetized (dashed line) animals. The cortex was illuminated by red light $(605 \mathrm{~nm})$, which is sensitive to changes in the concentration of deoxyhemoglobin. A downward deflection shows darkening of the cortex.

with a spatial frequency of $0.5-2$ cycles per degree). The control stimulus was a blank screen with a small $\left(0.1 \times 0.1^{\circ}\right)$ fixation point. To control for the physiological state of the cortex, the orientation maps and ocular dominance maps were obtained repeatedly from one patch of the striate cortex over a period of $>1$ year, as will be shown below.

To obtain functional maps from the optical imaging data, we followed the approach developed in previous work on anesthetized animals (Frostig et al., 1990; Malonek and Grinvald, 1996). There the concepts of "global signal" and "mapping signal" have been introduced and explained. In agreement with the definitions given there, the global signal was obtained by dividing the average response during all of the stimulus conditions by the pictures collected during the blank condition, whereas the mapping signal was obtained by dividing pictures collected during one stimulus condition by pictures collected during a different, "orthogonal," stimulus condition. The global signal is not colocalized with spiking activity in the imaged cortical area, whereas the mapping signal, usually much smaller, does colocalize with electrical activity and underlies the functional maps (Frostig et al., 1990; Grinvald et al., 1999).

Time courses of the global signal in awake monkeys and in anesthetized monkeys are shown in Figure 4. The $y$-axes represent the mean intensity of the reflected light from a selected part of the visual cortex during a period of stimulation divided by the mean intensity during a period of nonstimulation. The $x$-axes represent the time from stimulus onset. We found a large difference between the anesthetized and the behaving monkeys in the sizes of the global signals but not in the time course of the signals. Particularly, the initial dip amplitudes in the awake monkeys were up to threefold larger than in the anesthetized monkeys (the amplitude of the initial dip was $-0.003 \pm 0.001( \pm \mathrm{SE} ; n=15)$ in the awake monkeys and $-0.0015 \pm 0.0005( \pm \mathrm{SE} ; n=8)$ in the anesthetized monkeys. The relative amplitudes of the late component, known to originate from increased blood flow, appear even larger in the awake monkey.

Despite the large difference in amplitude, we found, however, that there was only a small difference in the time course of the signal between awake and anesthetized animals. In particular, the time of the initial dip in awake animals did not differ significantly from that in anesthetized animals $[2.3 \pm 0.5 \mathrm{sec}$ in awake monkeys $( \pm \mathrm{SE} ; n=15)$ and $2.0 \pm 0.3 \mathrm{sec}$ in anesthetized monkeys $(n=8)]$.

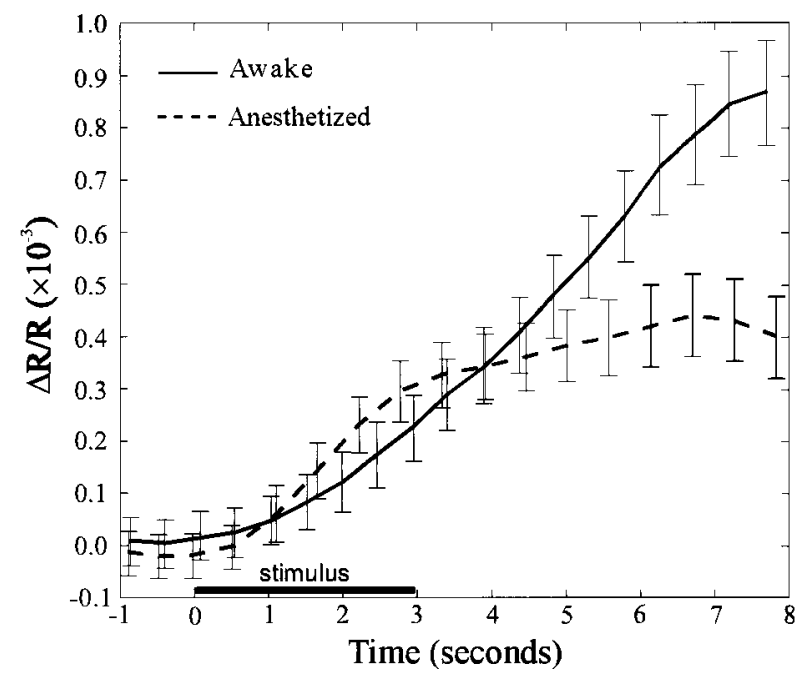

Figure 5. Time course of the mapping signal. Functional orientation maps were created by dividing (equivalent to subtraction) images of the cortex collected when the stimulus consisted of bars of $45^{\circ}$ by images collected when the stimulus consisted of bars of $135^{\circ}$. To show the time course of the map amplitude, the mean intensity of the centers of the black patches in the maps (patches of the cortex selective to bars of $45^{\circ}$ ) was then subtracted from the mean intensity of the white patches (patches of the cortex selective to bars of $135^{\circ}$ ) in each frame, and the result is plotted here as a function of time.

The time course of the mapping signal obtained from a series of differential images (using a pair of orthogonal stimuli) (Fig. 3C) is very different from the time course of the global signal. A time course of the mapping signals in awake (solid line) and anesthetized (dashed line) monkeys is plotted in Figure 5. There were only small differences in time course between awake and anesthetized monkeys during the first $7 \mathrm{sec}$. In a few experiments, when a longer recording period was used, we noticed that the mapping signals in the awake monkeys tended to decay more slowly than in the anesthetized monkeys. As with the global signals, there was a marked difference in the size of the mapping signals between awake and anesthetized monkeys; the mapping signal was up to threefold larger in the awake monkeys. As a measure for the strength of the mapping signal, we used the actual range of the map, referred to as the "clipping range," which is calculated by subtracting the intensity of the darkest feature in the functional map from that of the brightest feature and normalizing by the mean intensity. The mean clipping range for ocular dominance maps was significantly higher $(p<0.01)$ in the awake monkeys $[0.0035 \pm$ 0.0015 in the awake monkeys $( \pm \mathrm{SE} ; n=15)$ and $0.0011 \pm 0.0005$ in anesthetized monkeys $(n=8)]$. It is pertinent to mention in this respect that a large inter-animal variability was found in these parameters in both the anesthetized and the awake monkeys, whose origin remains to be explored. In addition in some of the behaving monkeys, the amplitude of the global and mapping signals decreased as a function of time (up to a factor of 2 in 1 year).

Although the mapping signal in the awake monkeys was up to threefold larger than in the anesthetized monkeys, the signal-tonoise ratio was not much larger because the noise level in the awake monkeys was twofold to threefold larger than in the anesthetized monkeys. One source of noise in awake monkeys is their movements, and by selecting only data collected when the monkey was quiet (sitting quietly in the chair with no movements except for small motor responses of the hand), we reduced the noise level by a factor of up to 2. Another major source of noise in the awake monkeys were eye movements. The contribution of eye movements to the noise level was estimated by comparing the orientation maps obtained when eye movements were within a narrow fixation window of $0.3 \times 0.3^{\circ}$ to the maps obtained when the window was $3 \times$ $3^{\circ}$. To exclude the possibility that the differences were attributable to body movements that are correlated with eye movements, we 
Two months after dura removal

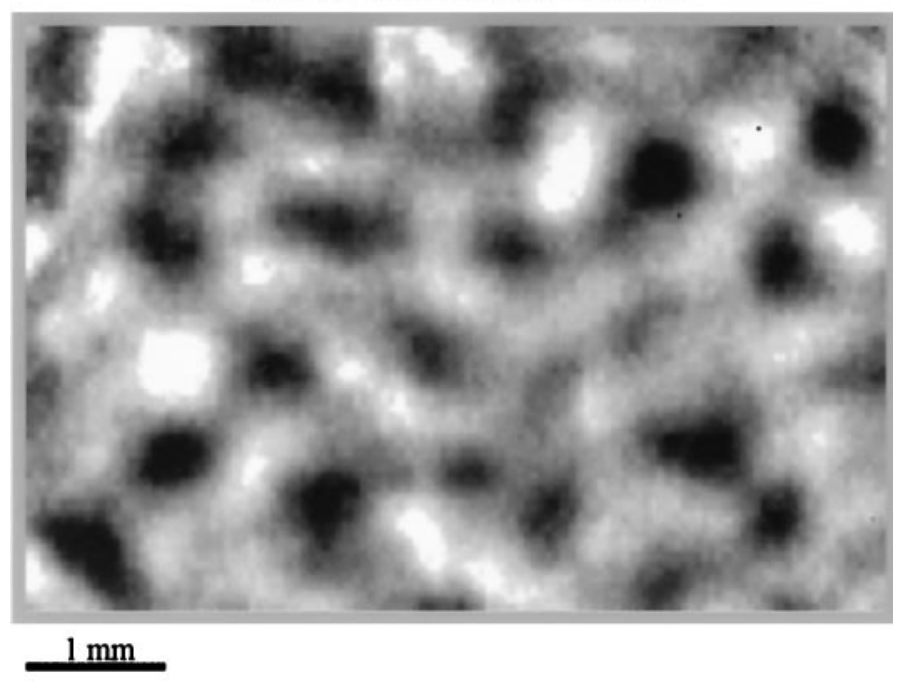

Eight months after dura removal

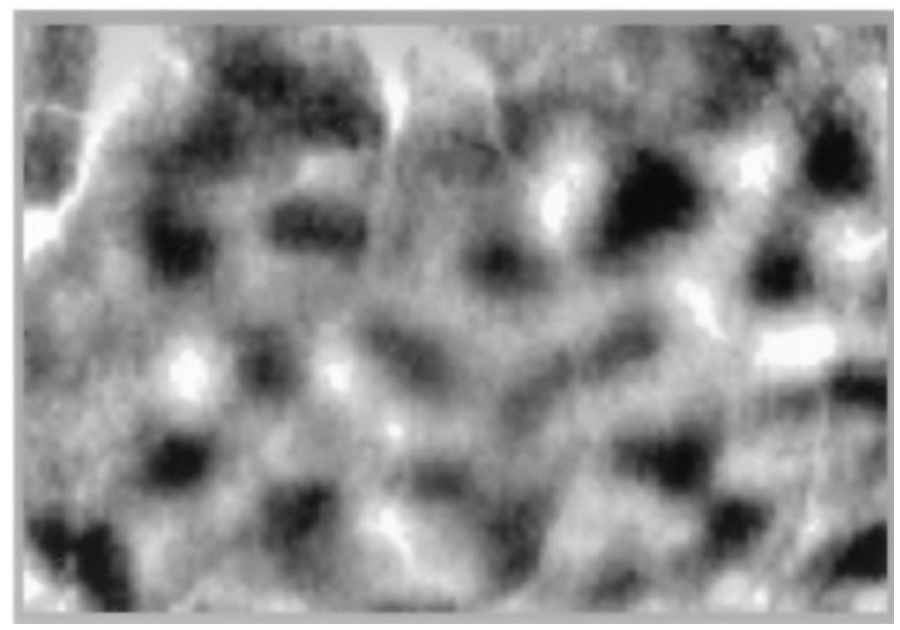

Figure 6. Two orientation maps collected from the same patch of the primary visual cortex, with a time difference of 6 months.

limited the comparison to periods in which the monkeys were sitting quietly. The noise level in the first case, when the monkeys were fixating tightly, was 1.5 - to 2-fold smaller than in the second case. We also compared the signal amplitude in both fixation conditions to find out how eye movements influence the raw signal. A small, barely significant increase in the signal amplitude was observed when the monkey fixates tightly [the amplitude of the initial dip increased from $-0.003 \pm 0.0005$ to $-0.0035(n=4)]$.

\section{Stability of functional maps}

To explore the stability of ocular dominance and orientation maps, we searched for significant changes in the functional maps over a period of up to 40 weeks. We used the vascular pattern seen in the raw images to find a linear transformation that would transform the images from each imaging session into a standard image with a given orientation and magnification. This transformation was then applied to the functional maps from the corresponding session. Figure 6 presents two orientation maps, obtained 6 months apart, after their transformation into the same coordinate system. The similarity between the maps is evident; all of the orientation domains that appear in the first map also appear in the second, and they also have similar shapes, indicating that the orientation maps did not change significantly over 6 months. A more elaborate comparison was performed with ocular dominance maps. These maps, obtained over 38 consecutive weeks, are displayed in Figure
7. The white lines, indicating the centers of the ocular dominance columns in the average map, are drawn at the same physical location (relative to the blood vessels) in all of the maps. It can be seen that there were no significant changes in the maps over 9 months. To emphasize the differences between the maps, the centers of the right-eye columns (Fig. 7, black columns) for each of the maps in Figure 7 are superimposed in Figure $8 A$. Again, the differences are small relative to the width of the columns.

To obtain a quantitative evaluation of the stability of the maps, we used a procedure similar to that described by Blasdel (1992) to find the centers of the ocular dominance columns. Briefly, the maps were bandpass filtered, and gradients maps were then produced. The zero crossing lines in the gradient maps were used as an objective estimate for the centers of the ocular dominance columns. The area between the average center of the ocular dominance columns and the centers calculated for each of the imaging sessions was taken as a measure of the changes in the maps (Fig. $8 B$ ). We termed this area the "difference area." The mean difference area over 9 months was $0.4 \pm 0.4 \mathrm{~mm}^{2}( \pm \mathrm{SE} ; n=31)$. For comparison, the average size of a column in the imaged area was $5.2 \mathrm{~mm}^{2}$. We calculated the significance of this change by comparing it with the difference area calculated within one session $(0.2 \pm$ $0.2 ; n=8)$ and over a few consecutive days $(0.4 \pm 0.3 ; n=7)$. It turned out to be not significant ( $t$ test; $p<0.05 ; n=31$ ). The real changes in the maps are probably smaller, but the accuracy of our methods is limited by nonlinear changes occurring in the vascular pattern over areas of $8 \mathrm{~mm}^{2}$ or more. Indeed, when restricting the analysis to selected areas of $2-3 \mathrm{~mm}^{2}$, we found that the variability of the maps was much smaller $\left(0.1 \pm 0.1 \mathrm{~mm}^{2} ; n=31\right)$.

Although the difference area was not significantly different from zero, one may wonder whether there were consistent, slow monotonic changes in the maps that were below noise level. To examine monotonic changes, we plotted the difference area as a function of time and used linear regression to determine whether there was a consistent trend in the change (Fig. 9). The slope of the line was not significantly different from zero $(t$ test; $p<0.01)$, indicating that there was no consistent monotonic change in the maps. Here, we do not rule out, however, small fluctuations in the maps.

\section{Changes in the concentrations of oxyhemoglobin and deoxyhemoglobin in awake monkeys as measured from spectroscopic data}

Imaging spectroscopy, an approach described in detail previously (Malonek and Grinvald, 1996; Malonek et al., 1997), was used to compare the interactions of electrical activity with the microcirculation in the primary visual cortices of awake and anesthetized animals. The interactions were found to be qualitatively similar, but the amplitudes of the changes were larger in the awake animal. The activity-dependent changes in the concentrations of oxyhemoglobin and deoxyhemoglobin after a visual stimulus are plotted in Figure 10. The solid lines show the changes in awake monkeys, and the dashed lines are the changes observed in anesthetized cats (adapted from Malonek and Grinvald, 1996). During the first $3 \mathrm{sec}$ of the response, no significant differences between awake and anesthetized animals were observed. In both groups, there was a clear increase in the concentration of deoxyhemoglobin, which reached a peak at $2.2 \pm 0.5 \sec ( \pm \mathrm{SE} ; n=4)$ after stimulus onset.

The validity of the simple linear decomposition method we used to estimate the stimulus-evoked changes in oxyhemoglobin and deoxyhemoglobin concentration changes (Eq. 1) has been questioned (Mayhew et al., 1998, 1999), because the path length of light penetrating the cortex is wavelength-dependent. It has been suggested that repeating the analysis at different wavelength ranges would test the adequacy of the approximation used. Therefore, we repeated the calculation for two independent wavelength ranges: $530-585$ and 585-650 nm. The results are plotted in Figure 10, $C$ and $D$. There were no significant differences between the time course of changes in the oxyhemoglobin or deoxyhemoglobin concentrations measured in the ranges of 530-585 and 585-650 nm. Neither of these time courses differed significantly from the time 


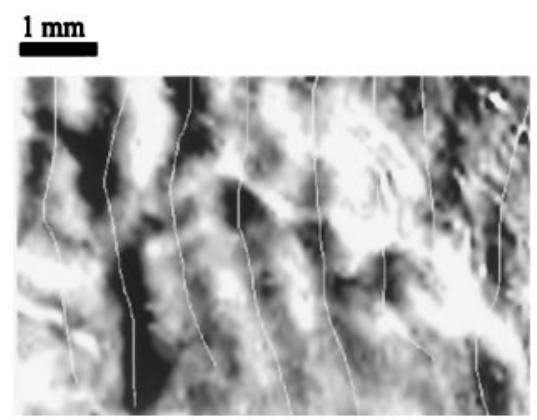

4 weeks

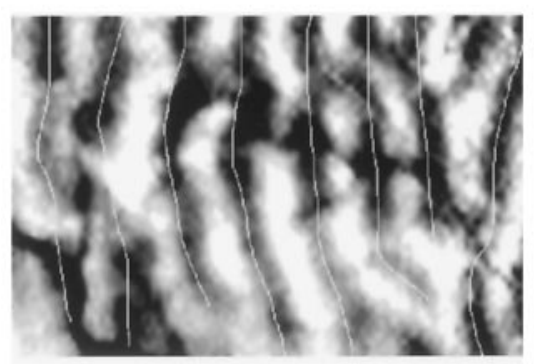

17 weeks

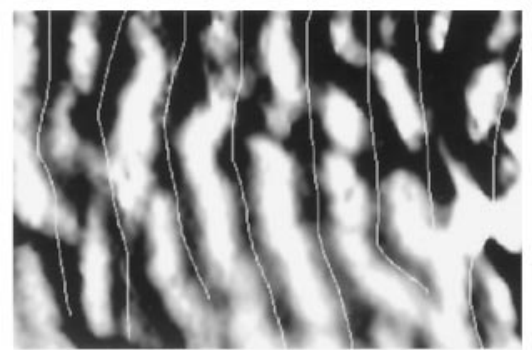

28 weeks

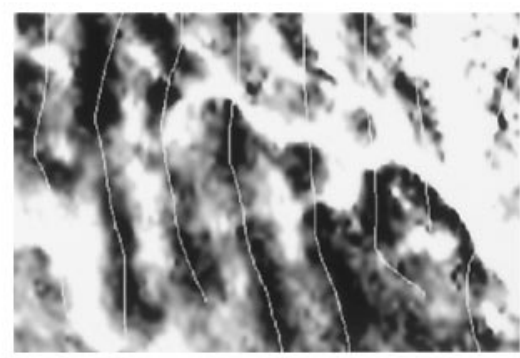

7 weeks

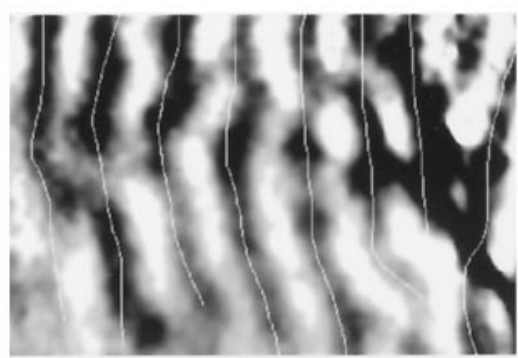

20 weeks

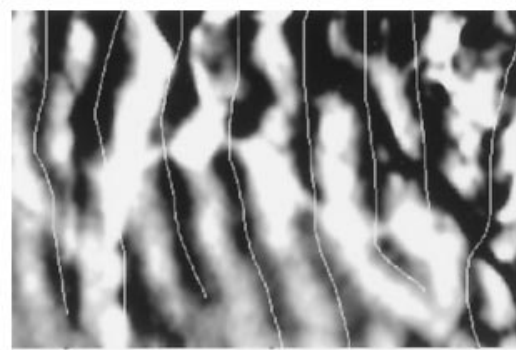

31 weeks

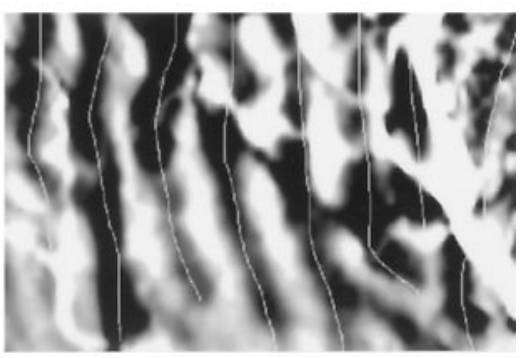

12 weeks

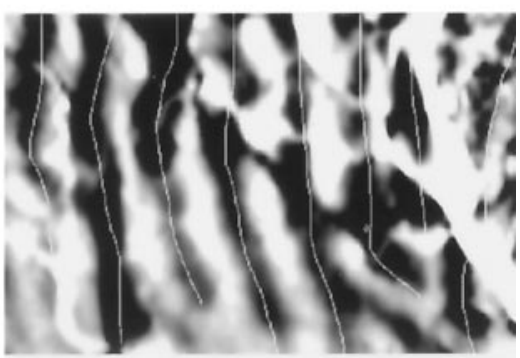

24 weeks

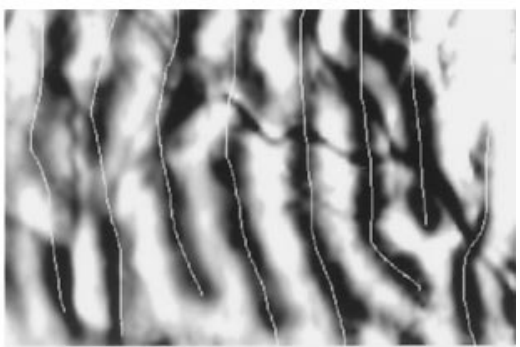

38 weeks

\section{Time from cortex exposure}

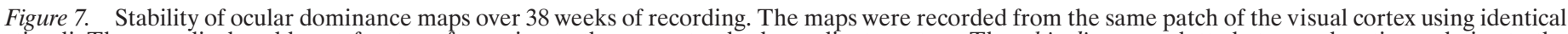

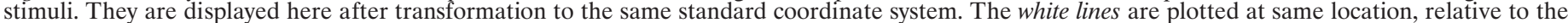
blood vessels, in all of the maps.

course calculated from the full spectral range of $530-650 \mathrm{~nm}$, suggesting that the simple linear approximation at this wavelength range did not introduce a large error into the time course thus obtained.

To estimate the individual contributions of the oxyhemoglobin and the deoxyhemoglobin concentrations to the functional maps, we linearly decomposed the spatiospectral ocular dominance maps into their oxyhemoglobin and deoxyhemoglobin components. The development of the maps with time in the awake monkey is shown in Figure $11 C$. The $x$-axes in these graphs represent time, and the $y$-axes represent space (see Materials and Methods). The ocular dominance columns are much sharper in the deoxyhemoglobin maps, indicating that the signals from the changes in concentration of deoxyhemoglobin are better colocalized with electrical activity than the signals from changes in oxyhemoglobin concentration. These results are qualitatively similar to those obtained previously in the anesthetized cat (Malonek and Grinvald, 1996).

To study the time course of the development of functional maps in the awake monkeys, we calculated the ratio between the mean mapping signal in all of the left-eye columns and in all of the right-eye columns (e.g., the mapping signal in the left-eye column is the ratio between the optical signal evoked by a left-eye stimulus to that evoked by a right-eye stimulus, in all of the pixels corresponding to the left-eye columns.). The time course of the mean ratio is plotted in Figure $11 C$. The mapping signal for deoxyhemoglobin appears earlier than for oxyhemoglobin. Both mapping signals increase monotonically during the first $8 \mathrm{sec}$ after stimulus onset. Whereas for the global signal the amplitude of the activitydependent changes in oxyhemoglobin were approximately sevenfold larger than that of deoxyhemoglobin (Fig. 10 A,B), the opposite amplitude relationship was found true for the amplitudes of the corresponding mapping signals (Fig. 11C). This difference in amplitudes is particularly large at early times; only after $\sim 10 \mathrm{sec}$ does the slower oxyhemoglobin mapping signal reaches a level similar to the level of the deoxyhemoglobin. Another difference between the global signal and the mapping signal is the lack of a significant initial peak in the time course of the deoxyhemoglobin mapping signal, similar to the clear peak observed in the time course of the 
A

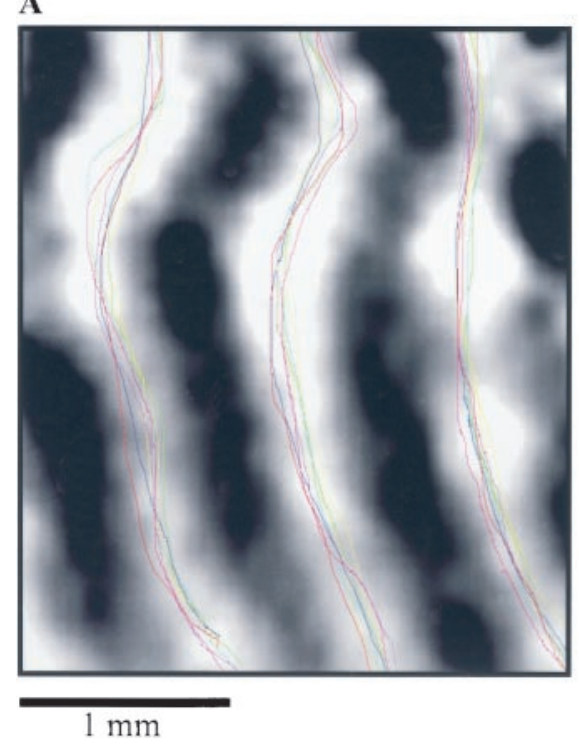

B

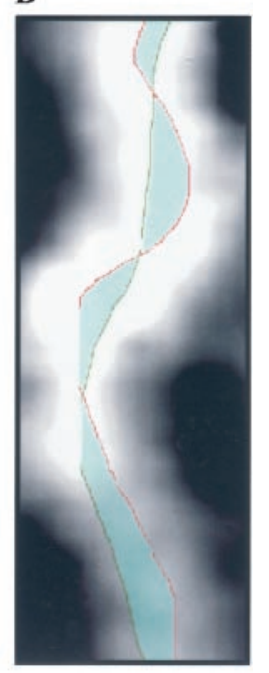

Figure 8. Changes in the centers of the right-eye ocular dominance columns over 38 weeks. $A$, Demonstration of the stability of three ocular dominance columns. The centers of the three columns were independently measured in nine different imaging sessions and then superimposed according to the blood vessel landmarks. $B$, Demonstration of the method of calculation of the difference area (blue). The green line is the center of a right-eye ocular dominance column measured on one of the recording days. The red line is the average value of the center of the column measured over several recording days. Scale bar, $1 \mathrm{~mm}$.

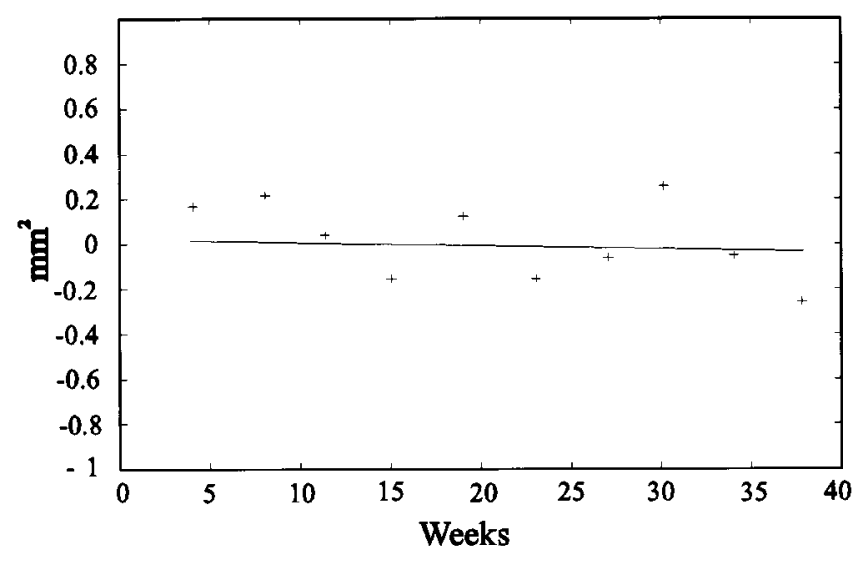

Figure 9. Mean difference area as a function of time from cortex exposure. Slow, consistent changes in the ocular dominance maps over a period of months would be indicated by a line with a slope different from zero. Linear regression analysis confirmed that the slope does not differ significantly from zero ( $t$ test; $p \leq 0.01$ ), indicating that there are no consistent changes in the location of the ocular dominance column centers.

global concentration of deoxyhemoglobin observed $\sim 3$ sec after stimulus onset (for comparison, see Fig. 10). Instead, one observes only a change in the slope of the mapping signal curve after the stimulus disappears (Fig. 11C). These observations can be explained by the following hypothesis: the initial increase in deoxyhemoglobin is a result of a fast, local increase in oxygen consumption during the stimulus presentation, whereas the large but delayed increase in oxyhemoglobin and the large decrease in deoxyhemoglobin $3 \mathrm{sec}$ after stimulus onset is caused by increased inflow of highly oxygenated blood (Fig. 10). This late blood flow increase is not as local as the increase in oxygen consumption because it is not well regulated at the level of cortical columns. Thus, the time course is not very different for two orthogonal stimuli. Because the mapping signal is obtained by subtraction of results obtained by two orthogonal stimuli from each other, the dip is not observed in the time course of the mapping signal, reflecting mainly changes caused by the local oxygen consumption.

\section{DISCUSSION}

The development of systems and methods for optical imaging from an awake animal over a time period of months enabled us to make a detailed comparison between the visual cortex of the anesthetized monkey and the visual cortex of the behaving monkey with respect to optical signals, functional maps, and the interaction between electrical activity and the cortical microcirculation. These developments facilitated the finding that, under normal conditions, ocular dominance columns and orientation columns are stable for a period of at least 1 year.

\section{Time course of the optical signals in awake, behaving monkeys}

Whereas the amplitude of the various components of the intrinsic signal was larger in the awake monkey, large differences were not found between the time course of the optical signals in awake and anesthetized monkeys. In both cases, there is a clear peak in the absorption of red light (wavelength of $600 \mathrm{~nm}$ or more) 2-3 sec after stimulus onset. The spectroscopic measurements (Fig. 10) supported the hypothesis that this peak is attributable to an increased oxygen consumption that causes an increase in the concentration of deoxyhemoglobin in the capillaries of the active cortical areas. Such an increase has not been detected, however, by most low-field strength f-MRI and positron emission tomography (PET) measurements, and it was therefore argued that the metabolism occurring immediately after a stimulus is mostly anaerobic. It might be thought that the discrepancy between the earlier optical spectroscopy studies and the f-MRI or PET studies can be explained by the differences in the subjects and the influence of anesthesia; all of the f-MRI and PET studies were done on awake human subjects, whereas all of the previous optical imaging and imaging spectroscopy studies were done on anesthetized animals. The present data show, however, that this is not the case, because neither the anesthesia nor the species difference affected the initial increase in the concentration of deoxyhemoglobin. It was suggested that the differences between the optical imaging and the f-MRI measurements might be a result of the lower sensitivity of f-MRI to rapid changes in deoxyhemoglobin concentration in the capillaries (Malonek and Grinvald, 1996; Vanzetta et al., 1999). This suggestion is now supported by a few f-MRI groups who observed the initial deoxyhemoglobin peak using high magnetic fields in humans (Menon et al., 1995; Hu et al., 1997; Yacoub and Hu, 1999). Furthermore, the initial dip was also clearly observed by $4.7 \mathrm{~T}$ f-MRI in the anesthetized monkey (Logothetis et al., 1999), suggesting that the relative amplitude of the initial dip is dependent on magnetic field strength rather than on species differences or anesthesia. This suggestion is in line with the theory underlying the relationship of the amplitude of the BOLD signal from capillaries and the magnetic field strength (Ogawa et al., 1993).

\section{The amplitude of the intrinsic optical signal in awake animals}

Both the global and the local intrinsic signals in awake monkeys are 1.5- to 3-fold stronger than in lightly anesthetized monkeys. These differences are not attributable to inter-animal or inter-session variance because they are significantly larger than these variances. Likewise, eye movements in the awake monkey could not explain these differences because the increase in signal amplitude induced by them is significantly smaller than the anesthetized awake differences. However, these differences do not seems to originate from the evoked electrical activity, because the observed ratio of evoked electrical response to spontaneous electrical activity in the visual cortex of awake animals is not significantly different from the ratio in lightly anesthetized animals (Livingstone and Hubel, 1981; Lamme et al., 1998). The most likely reasons for the weaker intrinsic signals in anesthetized monkeys is a weaker coupling 
A

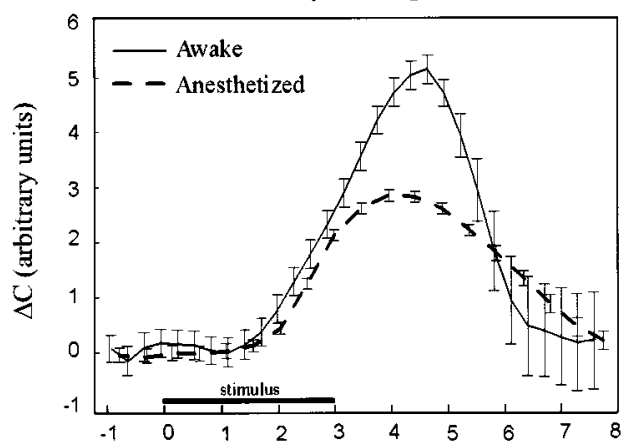

$\mathrm{C}$

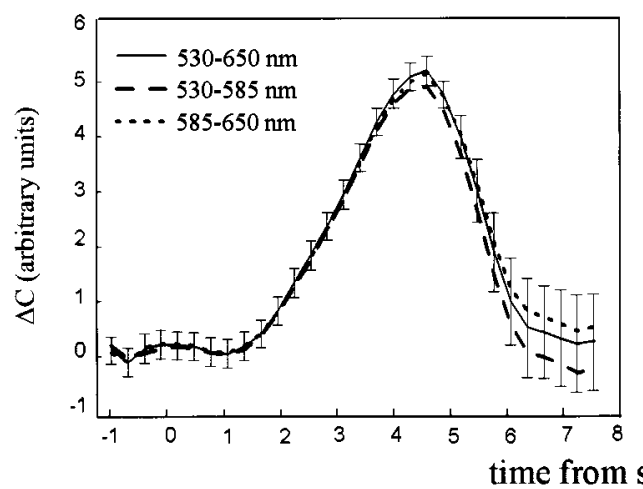

B Deoxyhemoglobin

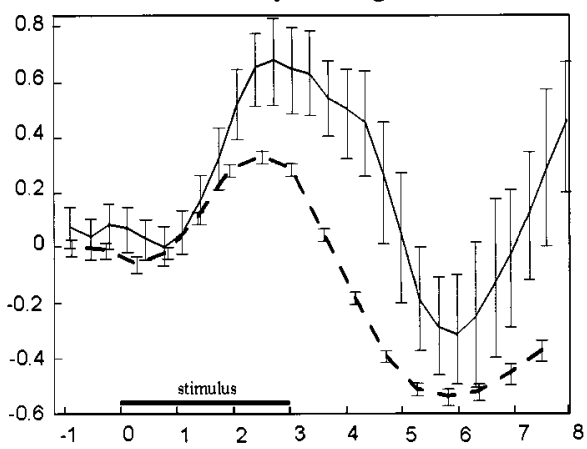

D

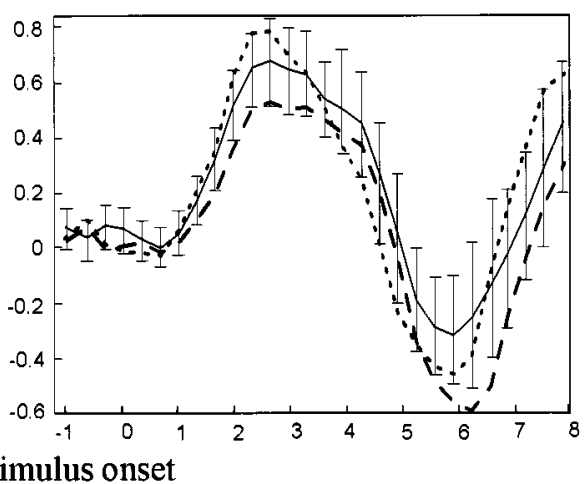

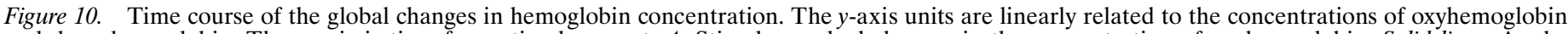

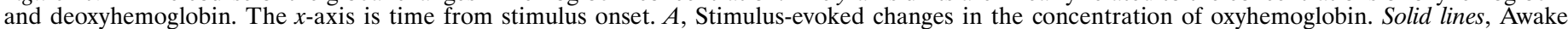

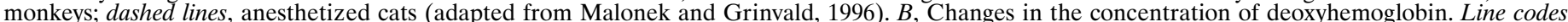

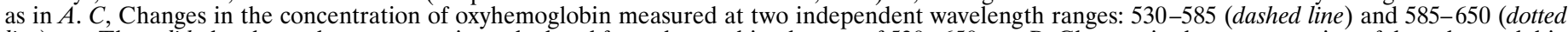

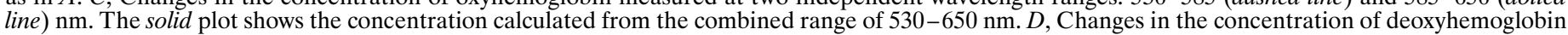
measured at two independent wavelength ranges. Line codes as in $C$.

\section{A Oxyhemoglobin map}

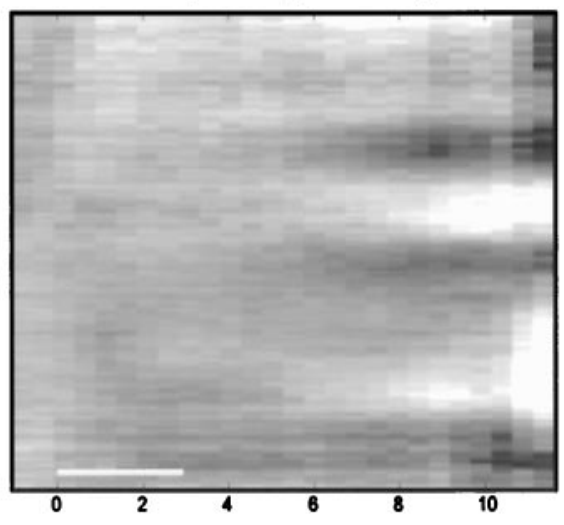

\section{B Deoxyhemoglobin map}

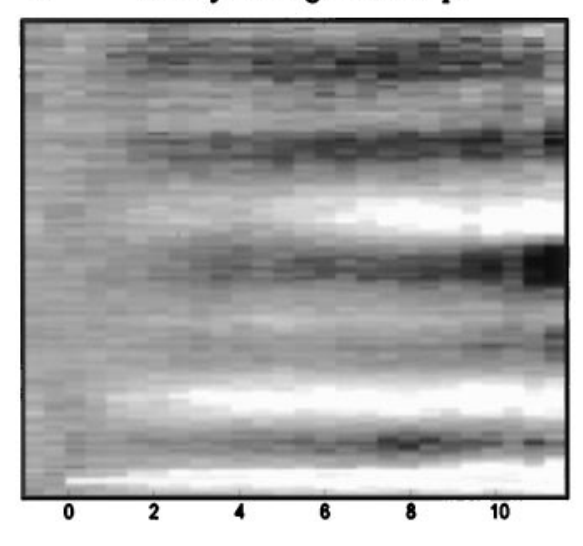

\section{C}

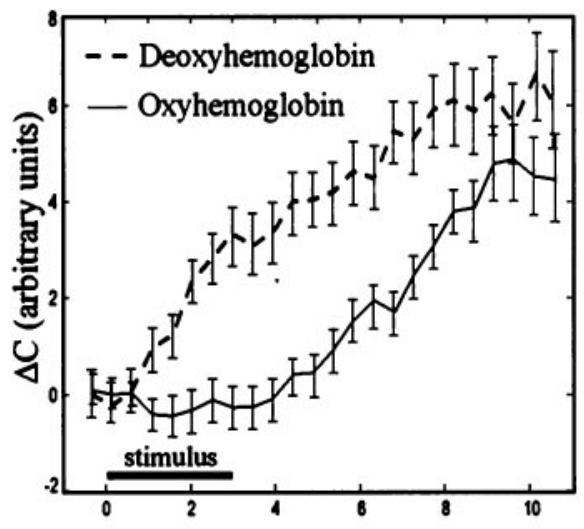

Seconds from stimulus onset

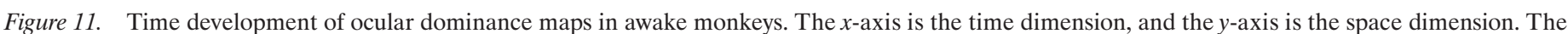

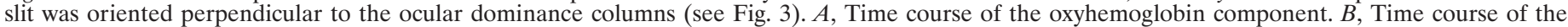

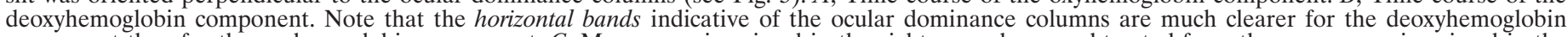

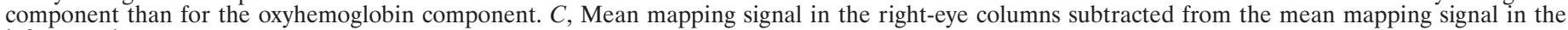
left-eye columns.

between neuronal activity and blood microcirculation attributable to anesthesia. In fact, this is a well known phenomenon observed during PET and f-MRI studies. This possibility is supported by previous observations of our group (our unpublished observations) of differences in intensity of the intrinsic signals under different anesthetic drugs, such as isoflurane and thiopental sodium, even if the depth of anesthesia is similar (as judged from EEG). Further systematic studies of the coupling between electrical activity and the microcirculation are needed to clarify the effect of anesthesia on the various components of the intrinsic signal.

\section{Imaging spectroscopy}

As mentioned above, two possible sources of the discrepancy between the f-MRI measurements and the spectroscopic measurements were excluded in this study: species difference and the influence of anesthesia. Recently, another possibility was raised: 
use of the wrong model for curve fitting of the results obtained by imaging spectroscopy (Mayhew et al., 1998, 1999). We believe that, although being oversimplified from the theoretical point of view, the linear model we use, neglecting wavelength-dependent path length differences, is not less precise than the alternative, more rigorous model proposed. The fact that the same results were obtained by imaging spectroscopy at three different wavelength ranges $(530-650,530-585$, and 585-650 nm) supports this conclusion. Furthermore, to verify the results obtained by imaging spectroscopy, blood oxygen level in the microcirculation was measured by a more direct method in our laboratory using the oxygen probe Oxyphore RII (Rumsey et al., 1988). The results obtained (Vanzetta and Grinvald, 1999) were nearly identical to those obtained by imaging spectroscopy. The most likely reason for the absence of the initial dip in most f-MRI data are the low sensitivity of low magnetic field f-MRI to the changes in deoxyhemoglobin within the capillaries. This question will probably soon be clarified with the development of f-MRI for awake monkeys (Dubowitz et al., 1998; Stefanacci et al., 1998; Logothetis et al., 1999).

What is the optimal time window for obtaining high-resolution hemodynamic maps corresponding to electrical activity? The answer critically depends on whether differential maps (i.e., maps computed by dividing two different stimulus conditions) or singlecondition maps (i.e., maps computed by dividing a stimulus condition by no-stimulus condition) are required to answer a particular question. Figures 3, 5, and 11 show that differential functional maps can be obtained at all wavelengths and for a long time after the stimulus offset. Thus, all components of the hemodynamic signal are stimulus-specific to some extent. In differential mapping methods as those reported here, it is advantageous to average over long time periods $(\sim 10 \mathrm{sec})$ because, in many circumstances, the nonstimulus-specific (global) part of the signal is cancelled out when the differential maps are obtained. When, however, it becomes necessary to map stimulus condition against no-stimulus condition (blank), one cannot get rid of the global part of the signals anymore. Recently (Grinvald et al., 2000) (Fig. 2C), we showed that, although the activity-dependent mapping component of the intrinsic signals continues to grow for a long time, the vascular artifact noise often grows much more, toward later times. Therefore, the best single condition maps are usually obtained during the first 3-4 sec, i.e., during the initial dip. How relevant these findings are to f-MRI is still controversial (Grinvald et al. 2000; Kim et al. 2000a,b; Logothetis, 2000).

\section{Stability of functional maps}

It is widely accepted that, under normal conditions, the functional maps in adult animals are stable (whereas under abnormal conditions, such as amputation or enucleation, the maps could change) (for review, see Kaas, 1991). In many studies of plasticity, this stability of the maps is implicitly assumed. However, stability of the cortical functional maps has been demonstrated only for gross features, such as gross retinotopic or somatosensory organization (Merzenich et al., 1984; Kaas et al., 1990; Heinen and Skavenski, 1991) or for statistical attributes of the maps (Creutzfeldt and Heggelund, 1975; LeVay et al., 1980). Stability of the maps in the submillimeter range, which is the size of the basic functional features in the visual cortex, was shown only for periods of a few weeks and only in kittens and ferrets (Kim and Bonhoeffer, 1994; Chapman et al., 1996; Godecke and Bonhoeffer, 1996). In the present study, changes in both orientation maps and ocular dominance maps, if they existed at all, were less than one-sixth of the size of a basic functional feature (i.e., a single orientation domain or a single ocular column) over periods of a few months, thus supporting the assumption of cortical map stability in the adult primate under normal visual experience.

Remarkable progress has been made in studying higher brain function using noninvasive imaging techniques that can be used on the human brain. However, because many electrophysiological and anatomical research tools are not applicable to noninvasive studies of the human brain, it appears that there is no alternative to using the behaving monkey as a model. Here we showed that highresolution optical imaging in the behaving monkey preparation is feasible for long-term studies. Furthermore, we have already shown that electrical recording, microstimulation, and tracer injections can be readily and simultaneously combined with optical imaging (Ts'o et al., 1990; Grinvald et al., 1991, 1999; Malach et al., 1993). In addition, we showed recently that voltage-sensitive dyes can also be used for long-term imaging of cortical dynamics in the awake monkey (Slovin et al., 1999). This result indicates that the fast millisecond by millisecond cortical dynamics explored previously only in the anesthetized cat (Arieli et al., 1995, 1996; Tsodyks et al., 1999) can now be explored in the behaving monkey. This multidisciplinary approach should shed light on the molecular, cellular, and network studies of the basic mechanism underlying sensory perception and higher brain functions.

\section{REFERENCES}

Arieli A, Shoham D, Hildesheim R, Grinvald A (1995) Coherent spatiotemporal pattern of on-going activity revealed by real-time optical imaging coupled with single unit recording in the cat visual cortex. J Neurophysiol 73:2072-2093.

Arieli A, Sterkin A, Grinvald A, Aertsen A (1996) Dynamics of on-going activity: explanation of the large variability in evoked cortical responses. Science 273:1868-1871.

Bartfeld E, Grinvald A (1992) Relationships between orientationpreference pinwheels, cytochrome oxidase blobs, and ocular dominance columns in primate striate cortex. Proc Natl Acad Sci USA 89:11905-11909.

Blasdel GG (1992) Differential imaging of ocular dominance and orientation selectivity in monkey striate cortex. J Neurosci 12:3115-3138.

Bonhoeffer T, Grinvald A (1991) Iso-orientation domains in cat visual cortex are arranged in pinwheel-like patterns. Nature 353:429-431.

Bonhoeffer T, Grinvald A (1996) Optical imaging based on intrinsic signals: the methodology. In: Brain mapping: the methods (Toga AW, Mazziotta JC, eds), pp 55-97. San Diego: Academic.

Bosking. WH, Zhang Y, Schofield B, Fitzpatrick D (1997). Orientation selectivity and the arrangement of horizontal connections in tree shrew striate cortex. J Neurosci 17:2112-2127.

Chapman B, Stryker MP, Bonhoeffer T (1996) Development of orientation preference maps in ferret primary visual cortex. J Neurosci 16:6443-6453.

Creutzfeldt OD, Heggelund P (1975) Neural plasticity in visual cortex of adult cats after exposure to visual patterns. Science 188:1025-1027.

Dubowitz DJ, Chen DY, Atkinson DJ, Grieve KL, Gillikin B, Bradley WGJ, Andersen RA (1998) Functional magnetic resonance imaging in macaque cortex. NeuroReport 9:2213-2218.

Frostig RD, Lieke EE, Ts'o DY, Grinvald A (1990) Cortical functional architecture and local coupling between neuronal activity and the microcirculation revealed by in vivo high-resolution optical imaging of intrinsic signals. Proc Natl Acad Sci USA 87:6082-6086.

Godecke I, Bonhoeffer T (1996) Development of identical orientation maps for two eyes without common visual experience. Nature 379:251-254.

Grinvald A, Lieke E, Frostig RD, Gilbert CD, Wiesel TN (1986) Functional architecture of cortex revealed by optical imaging of intrinsic signals. Nature 324:361-364.

Grinvald A, Frostig RD, Siegel RM, Bartfeld E (1991) High-resolution optical imaging of functional brain architecture in the awake monkey. Proc Natl Acad Sci USA 88:11559-11563.

Grinvald A, Shoham D, Shmuel A, Glaser D, Vanzetta I, Shtoyerman E, Slovin H, Arieli A (1999) In vivo optical imaging of cortical architecture and dynamics. In: Modern techniques in neuroscience research (Windhorst U, Johansson H, eds), pp 893-969. New York: Springer

Grinvald A, Slovin H, Vanzetta I (2000) Non-invasive visualization of cortical columns by f-MRI. Nat Neurosci 3:105-107.

Heinen SJ, Skavenski AA (1991) Recovery of visual responses in foveal V1 neurons following bilateral foveal lesions in adult monkey. Exp Brain Res 83:670-674.

Hu X, Le TH, Ugurbil K (1997) Evaluation of the early response in fMRI in individual subjects using short stimulus duration. Magn Reson Med 37:877-884.

Hubel DH, Wiesel TN (1962) Receptive fields, binocular interaction and functional architecture in the cat's visual cortex. J Physiol (Lond) 160:106-154.

Hubel DH, Wiesel TN (1969) Anatomical demonstration of columns in the monkey striate cortex. Nature 221:747-750.

Hubener M, Shoham D, Grinvald A, Bonhoeffer T (1997) Spatial relationships among three columnar systems in cat area 17. J Neurosci 17:9270-9284

Kaas JH (1991) Plasticity of sensory and motor maps in adult mammals. Annu Rev Neurosci 14:137-167.

Kaas JH, Krubitzer LA, Chino YM, Langston AL, Polley EH, Blair N 
(1990) Reorganization of retinotopic cortical maps in adult mammals after lesions of the retina. Science 248:229-231.

Kim DS, Bonhoeffer T (1994) Reverse occlusion leads to a precise restoration of orientation preference maps in visual cortex. Nature 370: 370-372.

Kim DS, Duong TQ, Kim SG (2000a) High-resolution mapping of isoorientation columns by fMRI. Nat Neurosci 3:164-169.

Kim DS, Duong TQ, Kim SG (2000b) Reply to "Can current fMRI techniques reveal the micro-architecture of cortex?" Nat Neurosci 3:414.

Kwong KK, Belliveau JW, Chesler DA, Goldberg IE, Weisskoff RM, Poncelet BP, Kennedy DN, Hoppel BE, Cohen MS, Turner R, Chang HM, Brady T, Rosen BR (1992) Dynamic magnetic resonance imaging of human brain activity during primary sensory stimulation. Proc Natl Acad Sci USA 89:5675-5679.

Lamme VA, Zipser K, Spekreijse H (1998) Figure-ground activity in primary visual cortex is suppressed by anesthesia. Proc Natl Acad Sci USA 95:3263-3268.

LeVay S, Wiesel TN, Hubel DH (1980) The development of ocular dominance columns in normal and visually deprived monkeys. J Comp Neurol 191:1-51.

Livingstone MS, Hubel DH (1981) Effects of sleep and arousal on the processing of visual information in the cat. Nature 291:554-561.

Logothetis N (2000) Can current fMRI techniques reveal the microarchitecture of cortex? Nat Neurosci 3:413-414.

Logothetis NK, Guggenberger H, Peled S, Pauls J (1999) Functional imaging of the monkey brain. Nat Neurosci 2:555-562.

Malach R, Amir Y, Harel M, Grinvald A (1993) Relationship between intrinsic connections and functional architecture revealed by optical imaging and in vivo targeted biocytin injections in primate striate cortex. Proc Natl Acad Sci USA 90:10469-10473.

Malonek D, Grinvald A (1996) Interactions between electrical activity and cortical microcirculation revealed by imaging spectroscopy: implications for functional brain mapping. Science 272:551-554.

Malonek D, Dirnagl U, Lindauer U, Yamada K, Kanno I, Grinvald A (1997) Vascular imprints of neuronal activity: relationships between the dynamics of cortical blood flow, oxygenation, and volume changes following sensory stimulation. Proc Natl Acad Sci USA 94:14826-14831.

Masino SA, Frostig RD (1996) Quantitative long-term imaging of the functional representation of a whisker in rat barrel cortex. Proc Natl Acad Sci USA 93:4942-4947.

Mayhew J, Hu D, Zheng Y, Askew S, Hou Y, Berwick J, Coffey PJ, Brown N (1998) An evaluation of linear model analysis techniques for processing images of microcirculation activity. NeuroImage 7:49-71.

Mayhew J, Zheng Y, Hou Y, Vuksanovic B, Berwick J, Askew S, Coffey P (1999) Spectroscopic analysis of changes in remitted illumination: the response to increased neural activity in brain. NeuroImage 10:304-326.

Menon RS, Ogawa S, Hu X, Strupp JP, Anderson P, Ugurbil K (1995) BOLD based functional MRI at 4 Tesla includes a capillary bed contribution: echo-planar imaging correlates with previous optical imaging using intrinsic signals. Magn Reson Med 33:453-459.

Merzenich MM, Nelson RJ, Stryker MP, Cynader MS, Schoppmann A, Zook JM (1984) Somatosensory cortical map changes following digit amputation in adult monkeys. J Comp Neurol 224:591-605.

Mountcastle VB (1957) Modality and topographic properties of single neurons of cat's somatic sensory cortex. J Neurophysiol 20:408-434.
Ogawa S, Lee TM, Kay AR, Tank DW (1990) Brain magnetic resonance imaging with contrast dependent on blood oxygenation. Proc Natl Acad Sci USA 87:9868-9872.

Ogawa S, Tank DW, Menon R, Ellermann JM, Kim SG, Merkle H, Ugurbil $\mathrm{K}$ (1992) Intrinsic signal changes accompanying sensory stimulation: functional brain mapping with magnetic resonance imaging. Proc Natl Acad Sci USA 89:5951-5955.

Ogawa S, Menon R, Tank DW, Kim SG, Merkle H, Ellermann JM, Ugurbil K (1993) Functional brain mapping by blood oxygenation leveldependent contrast magnetic resonance imaging. A comparison of signal characteristics with a biophysical model. Biophys J 64:803-812.

Polley DB, Chen-Bee CH, Frostig RD (1999) Two directions of plasticity in the sensory deprived adult cortex. Neuron 24:623-637.

Ratzlaff EH, Grinvald A (1991) A tandem-lens epifluorescence macroscope: hundred-fold brightness advantage for wide-field imaging. J Neurosci Methods 36:127-137.

Rumsey WL, Vanderkooi JM, Wilson DF (1988) Imaging of phosphorescence: a novel method for measuring oxygen distribution in perfused tissue. Science 241:1649-1651.

Shmuel A, Grinvald A (1996) Functional organization for direction of motion and its relationship to orientation maps in cat area 18. J Neurosci 16:6945-6964.

Shoham D, Hubener M, Schulze S, Grinvald A, Bonhoeffer T (1997) Spatio-temporal frequency domains and their relation to cytochrome oxidase staining in cat visual cortex. Nature 385:529-533.

Shtoyerman E, Arieli A, Grinvald A (1995) Optical imaging of the primary visual cortex in behaving monkeys. Isr J Med Sci [Abstr] 31:766.

Shtoyerman E, Vanzetta I, Grinvald A (1998) Spatio-temporal characteristics of cortical microcirculation response to visual stimulation in the awake monkeys. Soc Neurosci Abstr 24:10.4.

Slovin H, Arieli A, Hildesheim R, Grinvald A (1999) Voltage-sensitive dye imaging in the behaving monkey, p 129. Fifth IBRO World Congress of Neuroscience Abstracts.

Stefanacci L, Reber P, Costanza J, Wong E, Buxton R, Zola S, Squire L, Albright T (1998) fMRI of monkey visual cortex. Neuron 20:1051-1057.

Ts'o DY, Frostig RD, Lieke EE, Grinvald A (1990) Functional organization of primate visual cortex revealed by high resolution optical imaging. Science 249:417-420.

Tsodyks M, Kenet T, Grinvald A, Arieli A (1999) The spontaneous activity of single cortical neuron depends the underlying global functional architecture. Science 286:1943-1946.

Vanzetta I, Grinvald A (1999) Increased cortical oxidative metabolism due to sensory stimulation: implications for functional brain imaging. Science 286:1555-1558.

Vnek N, Ramsden BM, Hung CP, Goldman-Rakic PS, Roe AW (1999) Optical imaging of functional domains in the cortex of the awake and behaving monkey. Proc Natl Acad Sci USA 96:4057-4060.

Wang G, Tanaka K, Tanifuji M (1996) Optical imaging of functional organization in the monkey inferotemporal cortex. Science 272: $1665-1668$.

Wilson NC, Gisolfi CV, Phillips MI (1978) Influence of EGTA on an exercise-induced elevation in the colonic temperature of the rat. Brain Res Bull 3:97-100.

Yacoub E, Hu X (1999) Detection of the early negative response in f-MRI at 1.5 Tesla. Magn Reson Med 41:1088-1092. 